\title{
Fulminant Laryngeal-tracheobronchial-pulmonary Aspergillosis: A Rare and Fatal Complication in Allogeneic Hematopoietic Stem Cell Transplantation Recipients
}

\author{
Tao Tao ${ }^{1}$, Ying-Hui Zhang ${ }^{2}$, Sheng-Li Xue ${ }^{3-5}$, De-Pei Wu ${ }^{3-5}$ and Feng Chen $^{3-5}$
}

\begin{abstract}
A 23-year-old man who had previously undergone allogeneic hematopoietic stem cell transplantation (alloHSCT) for severe aplastic anemia was diagnosed with invasive laryngeal-tracheobronchial-pulmonary aspergillosis after presenting with a persistent dry cough at six months post-transplantation based on the findings of laryngoscopy and fiberoptic bronchoscopy. A fiberoptic bronchoscope was used to remove the obstructive material from the patient's airway and posaconazole plus caspofungin were administered to successfully to treat the patient. Our report suggests that laryngoscopy and fiberoptic bronchoscopy should be considered as alternative approaches to the diagnosis and treatment of allo-HSCT recipients with persistent respiratory symptoms when invasive laryngeal aspergillosis and invasive tracheobronchial aspergillosis are suspected.
\end{abstract}

Key words: aspergillosis, larynx, tracheobronchial, HSCT

(Intern Med 56: 347-351, 2017)

(DOI: 10.2169/internalmedicine.56.7201)

\section{Introduction}

Invasive fungal disease (IFD) remains a major cause of morbidity and mortality after allogeneic hematopoietic stem cell transplantation (allo-HSCT) (1); aspergillosis is the most common IFD infection among HSCT recipients $(2,3)$. The most common form of disease due to Aspergillus species in immunocompromised patients is invasive pulmonary aspergillosis (IPA), which mainly involves the lung parenchyma (4), the development of invasive tracheobronchial aspergillosis (ITBA) and invasive laryngeal aspergillosis is rare; however, the development of fulminant ITBA is associated with a serious prognosis $(5,6)$. While there are a few reports on ITBA in immunocompromised or immunocompetent hosts $(7,8)$, laryngeal aspergillosis is extremely rare (9). Unfortunately, there is limited experience in the treatment of ITBA and laryngeal aspergillosis in allo-HSCT patients.

We herein report a case of invasive laryngeal- tracheobronchial-pulmonary aspergillosis in a Chinese alloHSCT patient who was successfully treated and a review of the literature on serious complications after HSCT.

\section{Case Report}

A 23-year-old man with a history of severe aplastic anemia (SAA) underwent bone marrow transplantation from his HLA-haploidentical mother in January 2014. The conditioning regimens consisted of busulphan cyclophosphamide and antithymocyte globulin (BUCY+ATG) (10). Cyclosporine A (CsA) and short-term methotrexate (MTX) plus mycophenolate mofetil (MMF) were used as prophylaxis against graftversus-host disease (GVHD) (11). Standard measures were adopted for the prevention of infectious complications, which included fluconazole for antifungal prophylaxis and acyclovir to prevent herpes-related infections. A hemogram revealed the reconstruction of granulocytes (ANC $>0.5 \times 10^{9}$ / L) on day +12 post-transplantation. The patient developed

\footnotetext{
${ }^{1}$ Department of Hematology, the Xinghai Hospital of Suzhou Industrial Park, China, ${ }^{2}$ Department of Radiology, the Xinghai Hospital of Suzhou Industrial Park, China, ${ }^{3}$ Department of Hematology, the First Affiliated Hospital of Soochow University, China, ${ }^{4}$ Hematopoietic Transplant Institute, Soochow University, China and ${ }^{5}$ Jiangsu Institute of Hematology, the First Affiliated Hospital of Soochow University, China Received for publication January 27, 2016; Accepted for publication May 18, 2016 Correspondence to Dr. Feng Chen, chenfeng@medmail.com.cn
} 

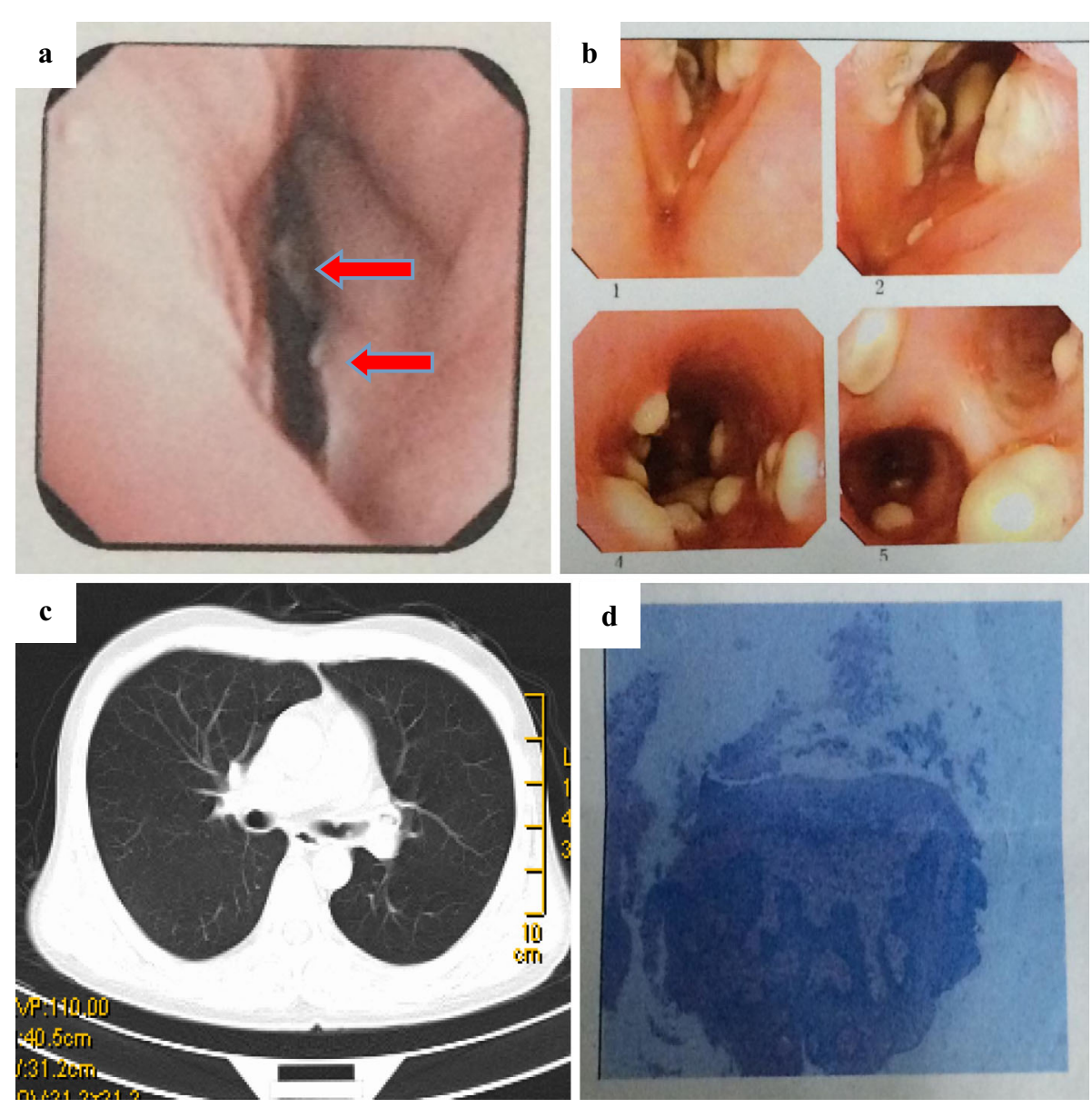

Figure 1. (a) Laryngoscopy revealed that the patient's throat was full of swollen ulcers with white plaque (marked by arrows). (b) Fiberoptic bronchoscopy demonstrated some irregular, nodular materials with white moss, which completely obstructed the bronchial wall. (c) Chest computed tomography (CT) revealed no specific findings. (d) A histopathological examination of the biopsy specimen revealed Aspergillus spp.

grade II acute GVHD of the skin on day +42 posttransplantation. This was treated by treatment with a standard-dose of methyl-prednisolone, which achieved a complete response (CR). The patient's chronic GVHD (cGVHD) of the skin gradually progressed from day +100 post-transplantation and he was treated with prednisolone and CsA. On day 120 post-transplantation, he complained of a cough and antibiotics were administered. A blood analysis revealed the following: WBC, $2.34 \times 10^{9} / \mathrm{L}$; ANC, $1.72 \times 10^{9} / \mathrm{L}$; hemoglobin, $85 \mathrm{~g} / \mathrm{L}$; and platelets, $72 \times 10^{9} / \mathrm{L}$. Although both a chest computed tomography (CT) scan and tests for pathogens via routine culturing, including blood tests for Beta-D glucan (G-test) and Galactomannan (GM-test) were all negative, the patient's cough did not respond to antibiotics and we empirically initiated treatment with voriconazole $(6 \mathrm{mg} /$ $\mathrm{kg} / 12 \mathrm{~h}$ for the first day, followed by $4 \mathrm{mg} / \mathrm{kg} / 12 \mathrm{~h}$ ). Liver toxicity occurred during voriconazole treatment, thus the anti-fungal regimen was changed to micafungin $(100 \mathrm{mg} / \mathrm{d})$. However, the persistent cough did not improve and hoarseness developed after two weeks of treatment - ulcers were then observed in the throat by laryngoscopy (Fig. 1a). The patient developed severe dyspnea in the following week when anti-infection and topical treatments were applied. Fiberoptic bronchoscopy revealed an irregular, nodular material with white moss, which nearly obstructed the bronchus; however, chest CT imaging was negative (Fig. $1 \mathrm{~b}$ and c). The histopathological examination of biopsy specimens revealed an Aspergillus species (Fig. 1d). The patient was diagnosed with pseudomembranous Aspergillus tracheobronchitis type ITBA based on the results of bronchoscopy and a pathological examination (12). The antibiotic and micafungin treatments were ceased and liposomal amphotericin B (liposomal AmB) was administered daily at a target dose of $3 \mathrm{mg} / \mathrm{kg}$. The patient's serum creatinine level rose from $60.4 \mu \mathrm{mol} / \mathrm{L}$ to $168 \mu \mathrm{mol} / \mathrm{L}$ during the first 7 days of liposomal AmB treatment. Due to progressive renal dysfunction, the anti-fungal regimen was switched to a combination of posaconazole $(400 \mathrm{mg} / 12 \mathrm{~h})$ and caspofungin [50 mg, daily (70 mg for the first dose)]. The combination therapy continued for 2 weeks, until the previous nodules in the throat completely disappeared under bronchoscopy (Fig. 2a); however, a repeat chest CT scan showed progression (Fig. 2b). The symptom of dyspnea gradually progressed, thus fiberoptic bronchoscopy was performed to remove the obstructive 

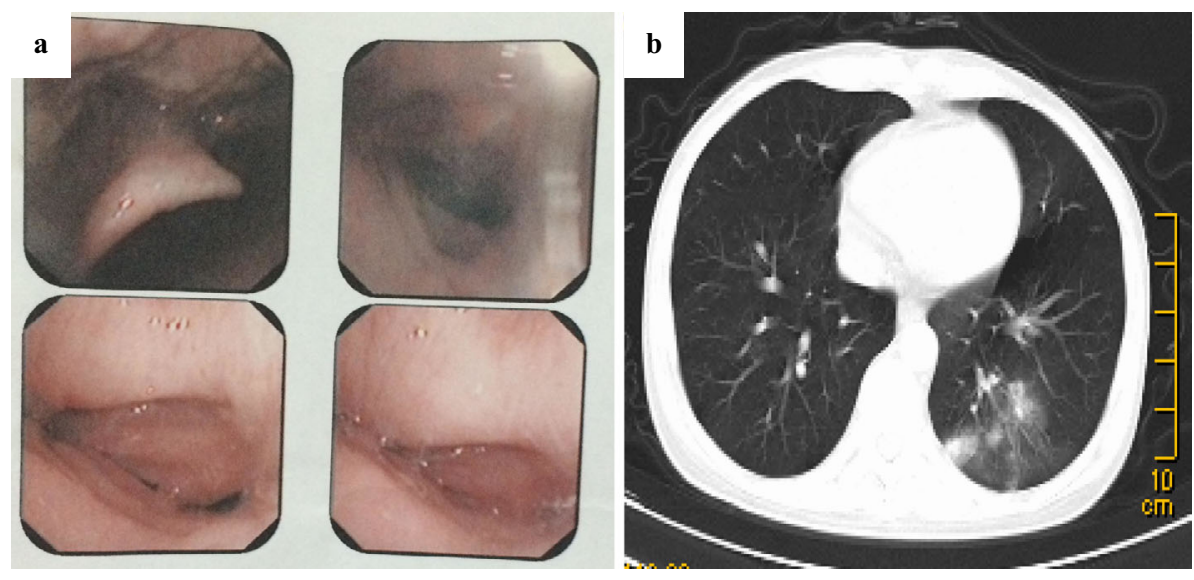

Figure 2. (a) The previous ulcers and nodules in the throat completely disappeared under bronchoscopy after the a combination anti-fungal therapy was administered for 2 weeks. (b) A repeat chest CT scan showed nonsegmental consolidation in the left lower lobe with the disappearance of the laryngeal lesions.

material from the patient's airways once a week for two weeks. All of the symptoms were relieved and the final chest CT scan showed negative results before the discontinuation of anti-fungal therapy, and all of the tests were negative for Aspergillus. Posaconazole was administered as a secondary prophylactic treatment and the patient was discharged from hospital. The patient is still being followed and remains free of any recurrence of invasive fungal infection.

\section{Discussion}

The latest multicenter study of the Organization for Research and Treatment of Cancer/Mycosis Study Group (EORTC/MSG) showed that the rate of invasive fungal infections (IFIs) among patients undergoing HSCT was lower than that in earlier studies (13). However, recent epidemiological studies in China have shown that the incidence of IFDs is increasing due to the increase in hematopoietic malignancies as well as the wide development of alloHSCTs $(14,15)$. The incidence of IFDs after haploidentical stem cell transplantation is significantly higher than that after HLA-matched sibling transplantation (2); Aspergillus is the most common pathogenic species of IFD in Chinese patients undergoing allo-HSCT (3). Patients with aGVHD and cGVHD, and elderly patients were reported to be at higher risk for fungal infections (16). ITBA and invasive laryngeal aspergillosis are rare clinical forms of IFD in which the Aspergillus infection is mainly limited to the tracheobronchial tree and larynx, and the incidence and the risk factors for these diseases are not fully defined in $\operatorname{HSCT}(17,18)$. Machida et al. (19) presented the case of a patient with ITBA after allo-HSCT together with a review of the literature. The prognosis of ITBA is significantly worse than that in organ transplant recipients due to the administration of immunosuppressive therapy and delayed immune reconstruction in HSCT patients. We would therefore like to add our recent experience, which shows that the larynx is an Aspergillussusceptible organ in allo-HSCTs recipients. Recently, we have successfully treated 3 cases of ITBA and/or invasive laryngeal aspergillosis in HSCT recipients, including the present case. Generally speaking, ITBA and invasive laryngeal aspergillosis should not be ignored as serious and potentially fatal complications after allo-HSCT.

Because of the low yield of cultures, the Food and Drug Administration (FDA) has approved the Aspergillus galactomannan and $\beta$-D-glucan assays as diagnostic tests for Aspergillus $(20,21)$. However, the tests are not useful for the early diagnosis of ITBA or invasive laryngeal aspergillosis, as their nonspecific clinical presentation and the sensitivity of the current tests are incompletely accurate (22). Persistent cough, dyspnea, hoarseness and fever were the most frequent symptoms observed in the pooled series of immunocompromised patients with ITBA and invasive laryngeal aspergillosis $(17,18)$. In the clinical setting, chest CT scan is still the first imaging modality to be applied in the initial diagnosis of ITBA. However, it is noteworthy that as the course of the infection progresses, radiologic abnormalities appear and that specific radiographic findings are not present in the early stages in these patients (23). According to the bronchoscopic appearance, there are two classifications of isolated ITBA that have been generally accepted $(12,24)$. Thus, numerous studies seem to confirm the point of view that these forms represent a progressive evolution of the disease, which ranges from mild to widespread bronchitis and are thus of educational rather than clinical value (25). The pseudomembranous and obstructive forms are most frequently observed in ITBA patients; the pseudomembranous type is usually found in severely immunocompromised hosts after HSCT (26). However, the manifestations of ITBA and invasive laryngeal aspergillosis under fiberoptic bronchoscopy and laryngoscopy, respectively, in HSCT patients have not been fully understood. The current patient was diagnosed with an Aspergillus infection by laryngoscopy and fi- 
beroptic bronchoscopy in a timely manner because he presented with a persistent cough at six months after transplantation. The rapid diagnosis and treatment of these complications improves survival after transplantation (27). Hence, we conclude that because the radiological signs are negative in the early disease period (when ITBA and/or invasive laryngeal aspergillosis were suspected), fiberoptic bronchoscopy and laryngoscopy should be employed as first-line investigations to obtain biopsy specimens and determine the etiology as soon as possible, and that thrombocytopenia or coagulopathy should be considered prior to obtaining a final diagnosis (28-30). In the present case, bronchoscopy revealed the presence of a large amount of obstructive material. Furthermore, the strong anti-fungal treatment did not improve the patient's dyspnea. The aggressive removal of the obstructive material (localized bronchial aspergillosis) using a fiberoptic bronchoscope was the key to the survival of this patient. Thus far, there is no consensus on the bronchoscopic or laryngoscopic management of allo-HSCT recipients and bronchoscopic removal has only been mentioned in a series of case reports $(31,32)$. The present case suggests that fiberoptic bronchoscopy should be considered as an alternative treatment option in patients with airway aspergillosis.

Voriconazole is the primary choice of medication for the treatment of Aspergillus (33, 34); however, we were forced to discontinue voriconazole treatment due to liver dysfunction. Micafungin has demonstrated in vitro and in vivo activity against Aspergillus spp. $(33,35)$; however, the obvious development of the obstructive material was observed in the airway under bronchoscopy during micafungin treatment in the present patient; which suggests that that Aspergillus may develop during micafungin therapy (36). Renal dysfunction is frequently observed in patients receiving liposomal AmB (37), which might have limited the use of this agent in the present patient. Several investigators have noted further improvements in the outcome based on in vitro and animal studies that have demonstrated the synergistic or additive effects of the combination of triazole or amphotericin B with echinocandin $(38,39)$. Combination antifungals should be considered for the treatment of invasive Aspergillus in the salvage setting (40). However, whether combination therapy is better than monotherapy remains to be determined (41). The present patient was treated successfully with a combination of posaconazole and caspofungin, demonstrating that the protocol is a feasible choice and that it should be considered as a salvage therapy (42).

In summary, ITBA and laryngeal aspergillosis should be considered as serious and potentially fatal complications after HSCT. Moreover, when ITBA and/or invasive laryngeal aspergillosis are suspected fiberoptic bronchoscopy and laryngoscopy should be performed as first-line investigations, as they allow for biopsy specimens to be rapidly obtained and for the etiology to be determined. Furthermore, fiberoptic bronchoscopy should be considered as an alternative treatment option in patients with airway aspergillosis. Finally, the results of the present case suggest that the combi- nation of posaconazole and caspofungin is a feasible treatment choice and that it should be considered as a salvage therapy.

The authors state that they have no Conflict of Interest (COI).

\section{Acknowledgement}

We appreciate Dr. Chun-Mei Ye, Dr. Jing Xu, Dr. Feng Du, Dr. Wei Gong and Dr. Yi-Shun Tan for their helpful work during the treatment of this patient.

Tao Tao, Ying-Hui Zhang, Sheng-Li Xue, De-Pei Wu and Feng Chen contributed equally to this work.

\section{References}

1. Pagano L, Caira M, Candoni A, et al. The epidemiology of fungal infections in patients with hematologic malignancies: the SEIFEM-2004 study. Haematologica 91: 1068-1075, 2006.

2. Sun Y, Xu L, Liu D, et al. Incidence of invasive fungal disease after unmanipulated haploidentical stem cell transplantation was significantly higher than that after HLA-matched sibling transplantation. Clin Microbiol Infect 19: 1029-1034, 2013.

3. Sun YQ, Xu LP, Liu DH, et al. The incidence and risk factors of invasive fungal infection after haploidentical haematopoietic stem cell transplantation without in vitro T-cell depletion. Clin Microbiol Infect 18: 997-1003, 2012.

4. Segal BH. Aspergillosis. N Engl J Med 360: 1870-1884, 2009.

5. Temime J, Mallat J, Van Grunderbeeck N, Gimenez S, Lemyze M. Fulminant invasive tracheobronchial aspergillosis. Am J Respir Crit Care Med 191: 848-849, 2015.

6. Williams MS, Ali N, Nonaka D, Bloor AJ, Somervaille TC. Fatal invasive aspergillosis of the larynx. Eur J Haematol 90: 354, 2013.

7. He H, Jiang S, Zhang L, et al. Aspergillus tracheobronchitis in critically ill patients with chronic obstructive pulmonary diseases. Mycoses 57: 473-482, 2014.

8. Hartemink KJ, Paul MA, Spijkstra JJ, Girbes AR, Polderman KH. Immunoparalysis as a cause for invasive aspergillosis? Intensive Care Med 29: 2068-2071, 2003.

9. Gangopadhyay M, Majumdar K, Bandyopadhyay A, Ghosh A. Invasive primary aspergillosis of the larynx presenting as hoarseness and a chronic nonhealing laryngeal ulcer in an immunocompetent host: a rare entity. Ear Nose Throat J 93: 265-268, 2014.

10. Liu DH, Xu LP, Zhang XH, et al. Substitution of cyclophosphamide in the modified BuCy regimen with fludarabine is associated with increased incidence of severe pneumonia: a rospective, randomized study. Int J Hematol 98: 708-715, 2013.

11. Storb R, Antin JH, Cutler C. Should methotrexate plus calcineurin inhibitors be considered standard of care for prophylaxis of acute graft-versus-host disease? Biol Blood Marrow Transplant 16: S18S27, 2010.

12. Kramer MR, Denning DW, Marshall SE, et al. Ulcerative tracheobronchitis after lung transplantation: a new form of invasive aspergillosis. Am Rev Respir Dis 144: 552-556, 1991.

13. Kurosawa M, Yonezumi M, Hashino S, et al. Epidemiology and treatment outcome of invasive fungal infections in patients with hematological malignancies. Int J Hematol 96: 748-757, 2012.

14. Hu R, Jiang XY, Wu Y. Risk factors for invasive pulmonary fungal infection in patients with hematological malignancies not receiving hematopoietic stem cell transplant. Neoplasma 59: 669-675, 2012.

15. Zhang P, Jiang EL, Yang DL, et al. Risk factors and prognosis of invasive fungal infection in allogeneic stem cell transplantation recipients: a single-institution experience. Transpl Infect Dis 12: 
316-321, 2010.

16. Srinivasan A, Wang C, Srivastava DK, et al. Timeline, epidemiology, and risk factors for bacterial, fungal, and viral infections in children and adolescents after allogeneic hematopoietic stem cell transplantation. Biol Blood Marrow Transplant 19: 94-101, 2013.

17. Fernández-Ruiz M, Silva JT, San-Juan R, et al. Aspergillus tracheobronchitis: report of 8 cases and review of the literature. Medicine (Baltimore) 91: 261-273, 2012.

18. Ohashi T, Mizuta K, Kuze B, Aoki M, Ito Y. Invasive epiglottic aspergillosis: a case report and literature review. Auris Nasus Larynx 42: 501-504, 2015.

19. Machida U, Kami M, Kanda Y, et al. Aspergillus tracheobronchitis after allogeneic bone marrow transplantation. Bone Marrow Transplant 24: 1145-1149, 1999.

20. De Pauw B, Walsh TJ, Donnelly JP, et al. Revised definitions of invasive fungal disease from the European organization for research and treatment of cancer/invasive fungal infections cooperative group and the national institute of allergy and infectious diseases mycoses study group (EORTC/MSG) consensus group. Clin Infect Dis Off Publ Infect Dis Soc Am 46: 1813-1821, 2008.

21. Guinea J, Bouza E. Current challenges in the microbiological diagnosis of invasive aspergillosis. Mycopathologia 178: 403-416, 2014.

22. Karageorgopoulos DE, Vouloumanou EK, Ntziora F, Michalopoulos A, Rafaildis PI, Falagas ME. $\beta$-D-glucan assay for the diagnosis of invasive fungal infections: a meta-analysis. Clin Infect Dis 52: 750-770, 2011.

23. Nishiyama KH, Falcão Ede A, Kay FU, Teles GB, Bernardi Fdel C, Funari MB. Acute tracheobronchitis caused by Aspergillus: case report and imaging findings. Radiol Bras 47: 317-319, 2014.

24. Wu N, Huang Y, Li Q, Bai C, Huang HD, Yao XP. Isolated invasive Aspergillus tracheobronchitis: a clinical study of 19 cases. Clin Microbiol Infect 16: 689-695, 2010.

25. Krenke R, Grabczak EM. Tracheobronchial manifestations of Aspergillus infections. ScientificWorldJournal 11: 2310-2329, 2011.

26. Tasci S, Glasmacher A, Lentini S, et al. Pseudomembranous and obstructive Aspergillus tracheobronchitis - optimal diagnostic strategy and outcome. Mycoses 49: 37-42, 2006.

27. Salmeron G, Porcher R, Bergeron A, et al. Persistent poor longterm prognosis of allogeneic hematopoietic stem cell transplant recipients surviving invasive aspergillosis. Haematologica 97: 13571363, 2012

28. Schelenz S, Barnes RA, Barton RC, et al. British Society for Medical Mycology best practice recommendations for the diagnosis of serious fungal diseases. Lancet Infect Dis 15: 461-474, 2015.

29. Kim SW, Rhee CK, Kang HS, et al. Diagnostic value of bronchoscopy in patients with hematologic malignancy and pulmonary infiltrates. Ann Hematol 94: 153-159, 2015.

30. Chellapandian D, Lehrnbecher T, Phillips B, et al. Bronchoalveolar lavage and lung biopsy in patients with cancer and hematopoietic stem-cell transplantation recipients: a systematic review and meta-analysis. J Clin Oncol 33: 501-509, 2015.

31. Grosu HB, Bashoura L, Ost D, Ordonez NG, Faiz SA. Critical airway obstruction due to pseudomembranous Aspergillus tracheitis. Am J Respir Crit Care Med 190: 65-66, 2014.

32. Stather DR, Tremblay A, MacEachern P, et al. Bronchoscopic removal of a large intracavitary pulmonary aspergilloma. Chest $\mathbf{1 4 3}$ : 238-241, 2013.

33. Walsh TJ, Anaissie EJ, Denning DW, et al. Treatment of aspergillosis: clinical practice guidelines of the Infectious Diseases Society of America. Clin Infect Dis 46: 327-360, 2008.

34. Herbrecht R, Denning DW, Patterson TF, Bennett JE, Greene RE, Oestmann JW. Voriconazole versus amphotericin B for primary therapy of invasive aspergillosis. N Engl J Med 347: 408-415, 2002.

35. Enoch DA, Idris SF, Aliyu SH, Micallef C, Sule O, Karas JA. Micafungin for the treatment of invasive aspergillosis. J Infect $\mathbf{6 8}$ : 507-526, 2014.

36. Imhof A, Balajee SA, Fredricks DN, Englund JA, Marr KA. Breakthrough fungal infections in stem cell transplant recipients receiving voriconazole. Clin Infect Dis 39: 743-746, 2004.

37. Wade RL, Chaudhari P, Natoli JL, Taylor RJ, Nathanson BH, Horn DL. Nephrotoxicity and other adverse events among inpatients receiving liposomal amphotericin B or amphotericin B lipid complex. Diagn Microbiol Infect Dis 76: 361-367, 2013.

38. Kirkpatrick WR, Perea S, Coco BJ, Patterson TF. Efficacy of caspofungin alone and in combination with voriconazole in a guinea pig model of invasive aspergillosis. Antimicrob Agents Chemother 46: 2564-2568, 2002.

39. Nagasaki Y, Eriguchi Y, Uchida Y, et al. Combination therapy with micafungin and amphotericin B for invasive pulmonary aspergillosis in an immunocompromised mouse model. J Antimicrob Chemother 64: 379-382, 2009.

40. Panackal AA, Parisini E, Proschan M. Salvage combination antifungal therapy for acute invasive aspergillosis may improve outcomes: a systematic review and meta-analysis. Int J Infect Dis 28: 80-94, 2014.

41. Marr KA, Schlamm H, Rottinghaus ST, et al. A randomised, double-blind study of combination antifungal therapy with voriconazole and anidulafungin versus voriconazole monotherapy for primary treatment of invasive aspergillosis. Clin Microbiol Infect 18: 713, 2012.

42. Raad II, Hanna HA, Boktour M, et al. Novel antifungal agents as salvage therapy for invasive aspergillosis in patients with hematologic malignancies: posaconazole compared with high-dose lipid formulations of amphotericin $\mathrm{B}$ alone or in combination with caspofungin. Leukemia 22: 496-503, 2008.

The Internal Medicine is an Open Access article distributed under the Creative Commons Attribution-NonCommercial-NoDerivatives 4.0 International License. To view the details of this license, please visit (https://creativecommons.org/licenses/ by-nc-nd/4.0/).

(C) 2017 The Japanese Society of Internal Medicine http://www.naika.or.jp/imonline/index.html 